\title{
Urgences
}

\section{Edipe recommencé d'Hubert Aquin : chantefable, centon, palimpseste}

\section{Renald Bérubé}

Numéro 24, juillet 1989

Le manuscrit sous l'angle

URI : https://id.erudit.org/iderudit/025534ar

DOI : https://doi.org/10.7202/025534ar

Aller au sommaire du numéro

Éditeur(s)

Urgences

ISSN

0226-9554 (imprimé)

1927-3924 (numérique)

Découvrir la revue

Citer cet article

Bérubé, R. (1989). Edipe recommencé d'Hubert Aquin : chantefable, centon, palimpseste. Urgences, (24), 77-88. https://doi.org/10.7202/025534ar d'utilisation que vous pouvez consulter en ligne.

https://apropos.erudit.org/fr/usagers/politique-dutilisation/ 


\section{CEdipe recommencé d'Hubert Aquin: chantefable, centon, palimpseste}

Renald Bérubé, Université du Québec à Rimouskı

A Louis-Georges Carrier

Projet de téléthéâtre: ce que je découvre, de plus en plus, c'est que tous les sujets que j'invente sont modelés sur des archétypes de situation dramatique et pourraient se classer dans une catégorie donnée de l'anthropologie dramatique sans plus de difficulté qu'une carte perforée fait son chemin dans un classeur. $\mathrm{Ce}_{\theta}$ déterminisme culturel qui pèse sur moi de tous ses antécédents m'inhibe et me subjugue à la fois. Dois-je baisser la télé et continuer modestement mon métier d'écrivain, c'est-àdire d'adaptateur de vieux thèmes? Ou bien dois-je me révolter et me dire que je vais sauver le déterminisme archétypal du thème par la forme artistique? [...] Dans un roman, je pourrais toujours profiter de cette polémique avec moi-même, mais au théâtre, à la TV? Je me vois m'engager dans des sujets qui m'apparaissent baignés dans le relativisme des situations ot des attitudes: j'acquiers une telle distance par rapport à mes histoires, que je les change à volonté, sans rien ressentir d'autre qu'une grande fatigue. J'ai le sentiment d'écrire des avariantes".

Hubert Aquin, Journal (inédit), 26 juillet 1961.

Sophocle faisant CEdipe roi avait déjà une légende sous les yeux et il n'a fait qu'une variante... [...] Sophocle signait une variante, pas un original, et il en est ainsi pour beaucoup d'auteurs.

Hubert Aquin, lors d'une interwiew, 1974. ${ }^{1}$

1 Yvon Boucher, «Aquin par Aquin» (interview), Le Québec littéraire, no 2 (no consacré entièrement à Aquin), Montréal, Guérin, 1976, p. 145. 
II sera sans doute un peu de mauvaise guerre, dans un colloque intitulé "Le manuscrit sous l'angle de la génétique littéraire", de commencer une communication en parlant de ce qui relève peut-être surtout de la genèse du texte inédit dont nous travaillons à l'édition critique. Mais il ne faut tout de même pas trop s'en faire: toute guerre étant probablement mauvaise, il devient dès lors de bon aloi de le souligner en commençant ainsi qu'originellement prévu.

II s'agira donc ici d'CEdipe recommencé, téléthéâtre écrit par Hubert Aquin en 1971. Téléthéâtre de 90 minutes, destiné à une réalisation radio-canadienne, le réalisateur devant être LouisGeorges Carrier, vieil ami et vieux complice d'Aquin, leur amitié/complicité remontant à l'époque de leurs études au collège Sainte-Marie durant la décennie 1940. Pour diverses raisons que nous évoquerons plus loin au fur et à mesure, ce téléthéâtre ne sera jamais réalisé, ne connaîtra pas sa destination finale, la réalisation et la télédiffusion. Mais on se doute bien, s'agissant d'un texte d'Aquin à l'occasion d'un colloque sur la génétique littéraire, que les choses, si elles s'arrêtent là, remontent à plus loin, à plus avant. D'où la nécessité du recours à la genèse, au roman des origines d'un téléthéâtre, roman des origines dont on sait, depuis Freud et Marthe Robert ${ }^{2}$, qu'il n'est jamais aussi simple qu'on pourrait le croire de prime abord.

Dans Point de fuite ${ }^{3}$, recueil de textes divers publié par Aquin en 1971 et dont le titre polysémique est fort bien choisi, on peut lire, en date du 10 avril 1970 dans la section "Lettres à Louis-Georges Carrier", un texte intitulé "PROJET (post mortem Amlödi)". II s'agit effectivement d'un post mortem en bonne et due forme: un projet de téléthéâtre Hamlet vient de prendre fin, sans avoir dépassé ce stade de projet. Pourtant, ledit projet a laissé d'autres traces: une lettre d'Aquin d'abord, datée du 22 mars 1970, et probablement destinée à Carrier. Lettre dans laquelle il décrit sommairement son projet et propose la date du 10 avril 1970 comme délai pour produire le texte; date qui, on vient de le voir, est aussi celle du post

2 Marthe Robert, Roman des origines et origines du roman [1972], coll. $\left.\propto T e\right|_{\infty}$, no 13, Paris, Gallimard,1976.

3 Hubert Aquin, Point de fuite, Montréal, Le cercle du livre de France, 1971. 
mortem. Mais surtout on retrouve, entre autres documents laissés par Aquin, un dossier de plus de cent feuillets intitulé "Hamlet (1970)" - et le premier feuillet, daté du 30 avril 1971, commence par le mot CEDIPE, suivi, deux lignes plus bas, du mot INSÉRENDES. Nous voilà donc avec un dossier "Hamlet (1970)" qui parle d'CEdipe et dont bien des feuillets sont datés de 1971, alors que d'autres sont intitulés, par exemple, "Insertions HA HAMLET 1970". Et il devient bien évident, pour qui a lu CEdipe recommencé, que le recueil de citations que constitue le dossier "Hamlet (1970)" a été largement utilisé par Aquin dans la composition de son téléthéâtre. Annotés de la main d'Aquin alors que les citations sont dactylographiées, certains feuillets indiquent ainsi que telle citation, disons de Nietzsche ou de Claudel, pourrait constituer une réplique de tel ou tel personnage de la pièce. Ce qui, selon les cas, adviendra ou n'adviendra pas; certaines citations sont aussi marquées en surimpression d'un $X$ manuscrit, après avoir effectivement été utilisées.

II y eut le projet Hamlet qui fit long feu, il y a le texte d'CEdipe recommencé: les premières manifestations du projet CEdipe datent des 10, 18 et 19 mars 1971, un an en gros après le projet Hamlet. La première se présente sous le titre "CEDIPEprojet HA-LGC, rencontre du 10 mars 1971". Aquin propose une "adaptation avec léger décentrage" qui, si l'on doit interpréter ses explications, utiliserait à peu près tous les procédés de la transtextualité: "juxtapositions de plusieurs connotations provenant d'autres héros"; “insérendes (cf: procédé ancien des copistes)"; «inclusion de variantes anciennes et modernes", "de considérations critiques (variantes au deuxième degré): Freud, Rank, Jung", etc. II demande de "gros moyens" à cause du rôle qu'il entend faire jouer au chœur, et il se fixe une échéance: le 10 avril 1971. Dans une autre entrée, il écrit que le "seul texte de HA" serait "celui du chœur" et explique comment pourrait se manifester ce dernier. II souligne également qu'il faut écrire ala sphinx (et non: le sphinx)" et indique que le "texte de base", c'est-à-dire pour nous l'hypotexte premier, sera "celui de Gide", nourri "d'insérendes provenant d'autres versions". Dans une troisième entrée, Aquin réaffirme que le texte de base sera celui d' "André Gide (CEdipe, 1930 - représenté par les Pitoëff en 32)", donne comme «datation" de l'œuvre le Ve siècle avant Jésus-Christ, en Grèce, discute de cachet et propose comme échéance le 15 avril 1971. II produira en effet un CEdipe daté du 26 avril 1971; 
et en dépit des intentions manifestées par Aquin, cette première version ne comporte pas de chœur et se déroule à une date non précisée mais contemporaine.

Deux éléments qu'il faut désormais retenir: que, dans un dossier intitulé "Hamlet-Notes + projets", Aquin écrivait, à l'entrée elle-même intitulée "CEdipe HA - Notes du 9 avril 1971": "Utiliser à mort les insertions prévues pour Hamlet!" Que le texte d'CEdipe recommencé est lui-même un réaménagement de son avant-texte, CEdipe, à partir des feuillets duquel il est écrit. Or cet avant-texte est annoté par Carrier, et Aquin, de toute évidence, a tenu compte des remarques de l'éventuel réalisateur de son texte. Le chœur est présent dans CEdipe recommencé, et telle scène, suggérée par Carrier, où le collier de Jocaste joue un rôle important, sera effectivement ajoutée. Ce qui n'est pas sans rappeler l'adaptation d'CEdipe faite par Louis-Georges Carrier lui-même et jouée à la salle du Gesù du Collège Sainte-Marie le 11 avril 1949 - Aquin tenait, dans cette adaptation, le róle de Créon. Et peut-être aura-t-on relevé, à travers les dates susmentionnées, que si CEdipe est daté du 26 avril 1971, le premier feuillet du recueil de citations "Hamlet (1970)" est daté, lui, du 30 avril 1971. Et les autres feuillets de cette même année sont datés, eux, de mai 1971. Ce qui pourrait impliquer que ces citations n'auraient été recueillies qu'en fonction de la version recommencée d' $\mathcal{E}$ dipe, ce que nous sommes à vérifier.

On aura compris, si cela qui précède est assez clair et atteint son but, que genèse et génétique, à un certain moment, ont tendance à se confondre. Le manuscrit/texte de base inédit sur lequel nous travaillons est lui-même le résultat d'une genèse (projet Hamlet qui se mue en projet $C$ Edipe) et d'une génétique (CEdipe recommencé est écrit à partir de son propre avant-texte aquinien, $C E d i p e)$ qui renvoie à l'CEdipe de Gide, aux annotations de Carrier et aux multiples lectures faites par Aquin et pour le projet Hamlet et pour le projet CEdipe. A quoi l'on pourrait ajouter, pour faire bonne ou mauvaise mesure, que Neige noire (1974), roman qui prend la forme d'un scénario et qui utilise beaucoup Hamlet, utilise aussi des citations qui se retrouvent dans le dossier "Hamlet (1970)".

Cela dit, qui se révèle certes complexe et qui ouvre de multiples pistes au lecteur/éditeur critique qui ne veut surtout 
pas faire abstraction de la génétique, acceptons maintenant de jouer le simple (?) jeu de la paratextualité et de mettre en regard l'un de l'autre le premier feuillet d'CEdipe recommencé et celui de son avant-texte, CEdipe. La première page d'CEdipe, si l'on fait abstraction des annotations de Carrier, se présente ainsi: en haut de la page, en majuscules soulignées, "CEDIPE”, suivi d'une parenthèse, "(titre de travail...?)". Quelques lignes plus bas, à gauche: "texte "original": Hubert Aquin"; et sous cette ligne, cette autre: "réalisation: Louis-Georges Carrier". Puis, plus bas à droite, la date du 26 avril 1971. Deux éléments à souligner: qu'CEdipe ne semblait pas encore un titre définitif, et que dès lors le CEdipe recommencé peut ne pas renvoyer au seul fait qu'il s'agissait là d'une version recommencée de son avant-texte. Que le mot "original", dans "texte "original'» est placé entre guillemets: Aquin indiquait déjà que son texte, par plusieurs de ses procédés, tenait aussi du palimpseste et de la variante. Le premier feuillet d'CEdipe recommencé, lui, sous le titre en capitales souligné plusieurs lignes plus bas que l'habitude le veut, contient une indication générique et le nom de l'auteur: "chantefable de Hubert Aquin". Plus bas et à gauche, entre parenthèses, l'indication "(90 minutes)". Plus bas encore et à droite, une date "1971", simplement suivie, quelques lignes plus bas, de: "Réalisation: Louis-Georges Carrier", en capitales. Ainsi, "CEDIPE (titre de travail...?) est devenu CEDIPE RECOMMENCE, et le "texte "original" une "chantefable". Et ce dernier mot vous renvoie à vos dictionnaires; Le grand Robert de 1965 ne le contenant pas, vous lisez dans Le petit Larousse illustré de 1973: "Récit médiéval mêlé de prose récitée et de vers chantés"; puis, dans Le nouveau Larousse classique de 1957: “Récit mêlé de prose et de vers $(\longrightarrow$ AUCASSIN ET NICOLETTE)". Et vous lisez sous Aucassin et Nicolette: "roman d'amour en prose et en vers (XIIIe s.), en forme de "chantefable"". Pour lire enfin, dans Le petit Robert de 1986, sous le vocable chantefable: "(XIIIe; de chanter, et fable). Récit médiéval en prose (récit) et vers (chant). Ex.: AUCASSIN ET NICOLETTE. - Poème de forme analogue. "Robert Desnos dont les chantefables [...] sont bien connues" (Bay)". Voulant en avoir le cœur net, édition critique et transtextualité ayant leurs droits, vous achetez Aucassin et Nicolette, pour constater que le texte est effectivement constitué, en alternance, de récits parlés/dialogués et de vers chantés. Et vous lisez le premier paragraphe de la préface à l'édition que vous avez achetée: 
Sans doute n'est-il pas rare que l'on ignore, comme pour Aucassin et Nicolette, par qui, où et quand a été écrite une œuvre du Moyen Âge qui est, pour une bonne part, le temps de l'anonymat. Mais d'autres problèmes, encore plus délicats, nous sont posés d'entrée de jeu. Aucassin et Nicolette est le seul échantillon du genre littéraire que l'on désigne par le mot de chantefable - ce terme n'apparaît d'ailleurs qu'une seule fois, précisément à la fin de notre texte - et où alternent, avec régularité, strophes assonancées et morceaux de prose: s'agit-il d'une œuvre unique dans tous les sens du mot, ou bien les autres se sont-elles perdues sans laisser de trace? ${ }^{4}$

"Version "originale" versus "chantefable": cela peut-il se lire "version "originale" versus version anonyme? II y aurait dès lors un paradoxe, figure que savait entre toutes pratiquer Aquin, puisque le générique "chantefable" est immédiatement suivi par "de Hubert Aquin" - une œuvre ne peut être à la fois anonyme et ne pas l'être. Et encore, paradoxe aidant... Bien sûr, le générique "chantefable" peut aussi renvoyer à sa manière, en court-circuitant les époques et les lieux ainsi que savent le faire les romans d'Aquin, à la construction même de la tragédie grecque dans laquelle atlernaient les parties dialoguées et les chants du chœur. Or précisément, après avoir été annoncé dès le projet initial sans s'être pourtant retrouvé dans CEdipe, le chœur est présent dans CEdipe recommencé. Mais peut-être la réponse à l'énigme, puisque le personnage d'CEdipe est en cause, peut-elle déjà se trouver plus loin dans le paratexte d'CEdipe recommencé, au carton 3 du "générique" que l'on peut bien considérer comme une épigraphe, et sur lequel on peut lire: ""Rien n'appartient à rien, tout appartient à tous..." Alfred de Musset (Namouna)". Si donc tout appartient à tous, cela est aussi mien que l'autre a écrit - dès lors, qui est l'auteur de

4 Aucassin et Nicolette (édition critique; $2^{\circ}$ édition revue et corrigée. Chronologie, préface, bibliographie, traduction et notes par Jean Dufournet), Paris, Garnier-Flammarion, $n^{\circ} 261,1984$, p. 8. Et vous lisez aussi, gràce à l'obligeance du collègue André Gervais, le recueil de Robert Desnos, Chantefables et Chantefleurs, Paris, Gründ, 1955. Pour constater qu'il s'agit de "petits poèmes pour les enfants" selon Youki Desnos qui les présente, petits poèmes aà chanter sur n'importe quel air» selon l'indication donnée sous le titre du recueil. Poèmes à chanter, mais qui peuvent aussi être simplement lus ou récités; le recueil de Desnos n'est pas une chantefable, récit à la fois dit et chanté, selon Aucassin et Nicolette. 
quoi, de quelle variante du déjà écrit suis-je l'auteur en quelque sorte anonyme?

On connaît assez bien les méthodes de travail d'Aquin. On pourrait dire de celles-ci ce que Raymonde Debray Genette dit de celles de Flaubert au moment où elle parle de «la réécriture des documents", "sorte particulière de genèse qui montre le chemin de l'endogenèse proprement dite":

Le cas de Flaubert est évidemment privilégié, puisque notre romancier était habité par la folie de la lecture préparatoire, et chose plus précieuse, par celle de la copie. Non seulement il nous a laissé des livres qu'il a consultés, mais des pages entières de arecopiages". [...] Certes, Flaubert lit pour se documenter, mais aussi et surtout par débauche imaginative, et ce sont les mots qui le débauchent. ${ }^{5}$

On croirait lire, ma foi, une description des pratiques aquiniennes: Aquin aimait dresser des listes de mots rares à utiliser - et en plus du recueil de citations déjà mentionné, il avait, pour la préparation de son Hamlet/CEdipe, colligé d'autres citations provenant de ses lectures hamléto-œdipiennes. II avait lu, si l'on peut dire, tous les "bons" livres sur le sujet: CEdipe et Hamlet d'Ernest Jones, Un œil en trop. Le complexe d'CEdipe dans la tragédie d'André Green, CEdipe ou la légende du conquérant de Marie Delcourt, la section "Hamlet" de l'Ulysse de Joyce ${ }^{6}$, et bien d'autres livres encore sur Sophocle, les théâtres grec et latin, sur l'inceste aussi, et diverses traductions de l'CEdipe roi. II s'était même dressé une liste des divers hypertextes d'CEdipe roi, liste qui contient jusqu'au CEdipe de Houdar de La Motte et à laquelle il ne manque, en gros, que l'CEdipe de Corneille.

Pourtant, et sauf le recueil de citations sur lequel nous reviendrons, Aquin utilise fort peu, somme toute, dans CEdipe

5 Raymonde Debray Genette, Métamorphoses du récit. Autour de Flaubert, coll. "Poétique», Paris, Seuil, 1988, p. 26-27.

6 Ernest Jones, Hamlet et CEdipe [1949 et 1967], coll. «Tel", n० 50, Paris, Gallimard, 1980; André Green, Un oil en trop. Le complexe d'CEdipe dans la tragédie [1969], Paris, Minuit, 1981; Marie Delcourt, CEdipe ou la légende du conquérant [1944], coll. a Confluents psychanalytiquesn, Paris, Les Belles Lettres, 1981; James Joyce, Ulysse. [1936 et 1937], coll. "Du monde entier», Paris, Gallimard, 1987. 
recommencé, les masses et les masses de documents recopiés. Un monde sépare le texte dernier, qui est de fait un texte encore inachevé, Edipe recommencé, des intentions d'Aquin telles qu'elles se révèlent dans les descriptions qu'il fait de son projet et des notes analytiques qu'il accumule et qu'il commente parfois d'un "TB" ou d'un "génial». Pour bien lire CEdipe recommencé, il faut non seulement le lire en regard d'CEdipe, son avant-texte, et des annotations de Carrier sur ce dernier, il faut encore le lire en regard du projet aquinien tel qu'il se révèle dans la masse de notes accumulées en vue de sa rédaction. Tous ces manuscrits, nous semble-t-il, doivent accompagner la publication d' Edipe recommencé - alors et alors seulement la genèse et la génétique peuvent donner toute leur mesure, alors et alors seulement peut-on suivre - voir aussi - les évolutions d'un projet et d'une écriture à l'œuvre.

Reste le recueil de citations intitulé "Hamlet (1970)", véritable petit CEdipe recommencé rêvé mais dont l'écriture du texte réussira mal à être à la hauteur. II s'agit donc de cela, d'un recueil de citations d'auteurs aussi multiples que divers, de Virgile à Dalida, en passant par Verlaine, Ramuz et Tzara. Citations fort souvent de seconde main, tirées d'études d'Albert Béguin, de Jean Rousset, de Marcel Raymond, de Maurice Blanchot. ${ }^{7}$ En gros et pour simplifier un peut trop peut-être, citations d'auteurs baroques d'une part, et romantiques allemands d'autre part; ce qui donne déjà une tonalité. Et de Shakespeare, "notre maître à tous" écrivait Aquin dès 1960, à l'occasion d'un projet TV Jules César. 8 Plusieurs de ces citations, cela a déjà été dit, se retrouvent sous forme de répliques de personnages ou du chœur dans CEdipe recommencé. Sans, bien sûr, que leur provenance soit identifiée. Quand, au feuillet 5 d'CEdipe recommencé, CEdipe dit: "Avant d'ouvrir les yeux, je sais tout par cœur", il dit une phrase de Claudel; quand, à la réplique suivante, il affirme: “Connaître,

7 Albert Béguin, L'âme romantique et le rêve [1939], Paris, José Corti, 1960; Jean Rousset, La littérature de l'âge baroque en France. Circé et le paon [1954], Paris, José Corti, 1968; Marcel Raymond, De Baudelaire au Surréalisme [1940], Paris, José Corti, 1963; Idem, Baroque \& renaissance poétique [1955], Paris, José Corti, 1964; Maurice Blanchot, Le livre à venir [1959], Paris, Gallimard, 1963.

8 Hubert Aquin, Point de fuite, p. 76. 
c'est se reporter en arrière; c'est par essence une régression infinie", il cite Nietzsche; et quand enfin, toujours au même feuillet, il répond ainsi à Jocaste: “Une durée infinie a précédé ma naissance... Qu'ai-je été pendant ce laps de temps... Vivre est perdre son temps; nous ne pouvons rien retrouver, ni rien garder...", il cite encore à son insu, Aquin seul sachant (et Carrier aussi), à la fois Schopenhauer et Santayana. Bien d'autres feuillets d'CEdipe recommencé ressemblent à ce feuillet 5, particulièrement lorsque Tirésias et le chœur prennent la parole.

Cette façon d'utiliser, doit-on dire anonymement, la pratique de la citation a un nom: le centon. Que Alain Duchesne et Thierry Leguay décrivent ainsi dans leur Petite fabrique de littérature:

Le centon peut être considéré comme une variante des activités de collage et de découpage.

Un peu oublié aujourd'hui (le dictionnaire Robert ne le mentionne pas) le centon est défini par Peignot comme aun morceau de poésie composé d'hémistiches de vers entlers ou de passages empruntés d'un ou de plusleurs auteurs. Le mot centon vient du grec, Kentrón (en latin cento), qul signifle habit fait de divers morceaux. Ce mot est formé de kentéó, piquer, parce qu'll fallait beaucoup de points d'algullle pour coudre ces sortes d'habits rapetassés. Ausone a prescrit des régles pour composer des centons. II faut prendre, dit-li, des morceaux détachés du même poète ou de plusieurs; on peut partager un vers, et en lier la moltía à une autre moltié prise allleurs, ou employer le vers tout entier; mais il n'est par permis d'Insérer deux vers suivis et pris dans le même endroit."

Et Peignot ajoute que ssouvent on étend la dénomination de centon aux ouvrages en prose, composés de morceaux dérobés. $\infty$

Pour résumer on peut dire que le centon consiste à découper des phrases dans des livres pour les recoller ensuite et composer un nouveau texte qul ne doit pas avoir de rapport - quant au contenu - avec les texte utilisés.

On parle ainsi d'une Vie de Jésus-Christ uniquement composée avec des passages de L'iliade et de L'odyssée d'Homère. ${ }^{9}$

9 Alain Duchesne et Thierry Leguay, Petite fabrique de littérature, Paris, Magnard, 1984, p. 97. 
Mon collègue André Gervais, à qui je dois la connaissance du livre de Duchesne et Leguay dont est extraite cette (longue) citation, me fait aussi remarquer qu'ainsi défini depuis son étymologie, le mot centon rejoint, dans sa signification figurée, celle de l' «habit d'Arlequin": "un tout formé de parties disparates", selon Le petit Robert. Ainsi, devant CEdipe recommencé, nous sommes en présence d'un CEdipe en habit d'Arlequin, théâtre ou téléthéâtre oblige, devant un CEdipe rapaillé qui peut nous envoyer à L'homme du même qualificatif selon Miron, ce dernier étant, nous le savons, notre Christ, selon Aquin, qui devait bien, comme d'habitude, parler en connaissance de cause.

Un autre exemple de feuillet-centon dans CEdipe recommencé? Le feuillet 47, qui fut d'abord numéroté 18-0:

\section{TIRÉSIAS}

La vérité est plus funeste que l'erreur...

GEDIPE

Qu'est-ce que cela veut dire...?

TIRÉSIAS

Refaire indéfiniment nous empèche d'inventer...

Encore une... énigme...?

CEDIPE

TIRÉSIAS

«Nel mezzo cammin di nostra vita

Mi ritrovai nella selva oscurra...»

Je ne comprends pas un mot...

CEDIPE

TIRESIAS

Avant de penser... il faut avoir inventé; avant de se souvenir, il faut avoir parcouru le chemin secret qui traverse la forêt obscure...

\section{CEDIPE}

(AU GREFFIER:) Dites à votre personnel de ne rien prendre en sténotypie...

\section{TIRÉSIAS}

Comme j'ai refusé de prêter serment, mes paroles, tout se passe, par raccourci, en hypothèses; j'évite de raconter...

Toutes les répliques de Tirésias, dans ce feuillet, sont entièrement ou partiellement empruntées: la première à Nietzsche, la seconde à Ramuz, la troisième à Dante (il s'agit du début de la Divine comédie), la quatrième à Nietzsche encore, la dernière à Mallarmé (cité par Blanchot). Et la plupart de ces 
emprunts se retrouvent dans le dossier "Hamlet (1970)", avec la référence - souvent donnée en abréviation, ce qui ne facilite certes pas la tâche de l'éditeur critique - à l'ouvrage où fut trouvée la citation.

Cela dit, il ne faudrait pas croire que tous les feuillets d'CEdipe recommencé ressemblent à celui-là que nous venons de présenter, bien au contraire; par ailleurs, il n'en demeure pas moins que le projet en lui-même relève du palimpseste, de l'hypertextualité, de la littérature au xième degré. CEdipe recommencé remonte au mythe d'CEdipe - dont on sait qu'il traverse l'œuvre d'Aquin depuis L'invention de la mort, roman inédit de 1959 - en passant par l'CEdipe d'Aquin lui-même annoté par Carrier, ce dernier étant aussi l'auteur d'un CEdipe dans lequel avait joué Aquin. Et il faut voir comment CEdipe recommencé se compose à partir d'CEdipe, son avant-texte: Aquin tient compte des remarques de Carrier, ajoute, biffe, rature - mais peut oublier, sur tel feuillet d'CEdipe devenu feuillet d'CEdipe recommencé, de réaménager tel passage pour tenir compte de tel réaménagement effectué antérieurement. Si bien qu'à la rigueur, CEdipe recommencé, mosaïque/montage inachevé, peut difficilement se lire sans son avant-texte. Ce qui explique que nous avons choisi, dans l'édition critique, d'éditer l'avant-texte et le texte en regard l'un de l'autre. Et idéalement, les dossiers du type "Hamlet (1970)" de même que l'CEdipe roi de Carrier (publié dans les... "Cahiers d'Arlequin" Iors de la création de la pièce au collège Sainte-Marie) devraient être publiés en annexe. Ainsi, l'édition critique et la génétique littéraire pourraient, psychanalyse oblige lorsqu'il est question d'CEdipe, réaliser une heureuse fusion.

Mais revenons à la pratique du centon utilisée à l'occasion d'une chantefable. Cette pratique, nous semble-t-il, peut se lire comme le stade ultime du palimpseste, comme la limite de l'hypertextualité, de la conscience de la variante. Et cette pratique est aussi responsable, entre autres raisons, du fait qu'CEdipe recommencé ne fut jamais ni réalisé, ni donc (!) télédiffusé, Carrier craignant des accusations de plagiat. ${ }^{10}$

10 Voir ce que dit Carrier dans le livre de Françoise Maccabée-lqbal, Desafinado. Otobiographie de Hubert Aquin, Montréal, VLB éditeur, 1987 , p. 307. 
Ainsi, CEdipe recommencé, dont le sujet est l'œdipe qui sans cesse recommence par-delà les âges et les lieux et dont la composition, en accord avec le sujet, renvoie à toutes les pratiques de la trans- ou de l'intertextualité, peut effectivement se lire selon son générique, "chantefable": il y a alternance du parlé et du chanté (comme dans la tragédie grecque), il y a aussi anonymat comme dans l'Aucassin et Nicolette médiéval. Anonymat des auteurs cités, qui parlaient donc d'CEdipe sans le savoir pourrait-on dire; anonymat d'Aquin, ó paradoxe, puisqu'il ne fait toujours que donner la parole aux autres pour donner forme, ó paradoxe encore, à son CEdipe. Ainsi se trouve réalisée, dans la polyphonie vocale, "la disparition élocutoire du poète"; ainsi se trouve affirmée sa présence. ${ }^{11}$

11 Hubert Aquin, «"la Disparition élocutoire du poète" (Mallarmé)» [1974], article repris dans le recueil Blocs erratiques, coll. aProse entière», Montréal, Quinze, 1977, p. 263-267. 